\title{
Articaine and lignocaine
}

\author{
Abstracted from \\ Katyal V.
}

The efficacy and safety of articaine versus lignocaine in dental treatments: a meta-analysis. J Dent 2010; 38: 307-317.

Address for correspondence; V Katyal, School of Public Health, University of Sydney, Sydney NSW 2006, Australia.

E-mail:vkat8650@uni.sydney.edu.au

\section{Question: What is the efficacy and safety} of articaine compared with lignocaine in maxillary and mandibular infiltrations and block anaesthesia in patients presenting for routine dental treatments?

Data sources Cochrane Central, Medline, Embase, and ProQuest Health and Medical Complete, the metaRegister of the controlled trials database and a leading manufacturer.

Study selection Randomised controlled trials in patients requiring non-complex routine dental treatments comparing $4 \%$ articaine (1:100,000 epinephrine) with $2 \%$ lignocaine (1:100,000 epinephrine) for maxillary and mandibular infiltrations and block anaesthesia were included. The principal outcome measures were anaesthetic success, onset of action, post-injection adverse events or post-injection pain. Data extraction and synthesis Following data abstraction a metaanalysis was performed using random-effects model.

Results Eight studies were included in a meta-analysis. Articaine is more likely than lignocaine to achieve an anaesthetic success in the posterior first molar area, with a relative risk for success of $1.31(95 \% \mathrm{Cl}$ $1.12-1.54, P=0.0009)$. There is no difference in post-injection adverse events between articaine and lignocaine with a relative risk of 1.05 (95\% $\mathrm{Cl} 0.66-1.65, \mathrm{P}=0.85)$. However, articaine injection results in a higher pain score as measured by Visual Analogue Scale than lignocaine at the injection site, after anaesthetic reversal, with a weighted mean difference of 6.49 (95\% $\mathrm{Cl} 0.02-12.96, \mathrm{P}=0.05)$ decreasing to 1.10 ( $95 \% \mathrm{Cl} 0.18-2.02, \mathrm{P}=0.02)$ on the third day after injection. Conclusions The results of this systematic review provide support for the argument that articaine is more effective than lignocaine in providing anaesthetic success in the first molar region for routine dental procedures. In addition, both drugs appear to have similar adverse effect profiles. The clinical impact of articaine's higher post-injection pain scores than lignocaine is negligible. Hence, articaine is a superior anaesthetic to lignocaine for use in routine dental procedures. Use in children under 4 years of age is not recommended, since no data exist to support such usage.

\section{Commentary}

Articaine became available as a dental local anaesthetic in the UK in 1998. Its introduction has led to two research questions being addressed. Firstly, is $4 \%$ articaine with adrenaline more effective than $2 \%$ lidocaine with adrenaline? Secondly, does the higher concentration increase the incidence of adverse effects with the former solution? It might seem simple to answer the efficacy question and a number of trials have investigated this. Similarly, a metaanalysis of the efficacy studies should provide useful information. Unfortunately it is not that easy. A major problem when investigating the efficacy of local anaesthetic solutions is a lack of a universally accepted outcome measure. Although the studies included in this meta-analysis considered anaesthetic success as an outcome measure, they did not employ a consistent definition of success. The inclusion criteria for this analysis mentions that subjects had to have non-complex dental treatment; however some of the studies incorporated were performed in volunteers having no dental treatment. Even in these volunteer studies, where anaesthesia was determined on the basis of no response to an electronic pulp tester, different definitions of success were used. One volunteer study ${ }^{1}$ defined successful anaesthesia as two consecutive non responses within 15 minutes sustained for 60 minutes. Another ${ }^{2}$ used two consecutive non responses at any time within 30 minutes as the criterion for success. So the outcome measures within the volunteer studies varied and this is compounded with the inclusion of investigations where some treatment was performed. In short the outcome measures were inconsistent within the incorporated studies. Another factor that may be of importance is the dose of local anaesthetic used. This varied between the investigations considered and unfortunately has not been accurately recorded in the summary table as the dose claimed to have been used in one of the studies ${ }^{3}$ was overestimated four-fold.

It is very important to point out that different injection techniques were compared in this analysis. This is relevant in relation to the question about adverse effects. As noted in the review ${ }^{4}$ there has been a suggestion that $4 \%$ articaine may produce more non-surgical paraesthesias compared to $2 \%$ lidocaine following mandibular block injections. So an important question to answer is "Does articaine offer any benefit in mandibular block anaesthesia?" It must be stressed that the risk of non-surgical paraesthesia is small. It is therefore important to determine if there is any advantage to the use of $4 \%$ articaine for mandibular blocks, as any increase in efficacy compared to $2 \%$ lidocaine could outweigh the low risk of paraesthesia. Unfortunately because of the multiple techniques compared in this 
meta-analysis such a question cannot be satisfactorily addressed. It appears that those studies included in the analysis that compared mandibular infiltration for the two drugs showed definite improvement in "success" with the former drug for the lower first molar in volunteers. It is possible that this improved efficacy of $4 \%$ articaine in the mandibular infiltration technique weighted the data to give an overall increased efficacy for this drug for the pooled data. So it is not possible to tell if $4 \%$ articaine is more effective than $2 \%$ lidocaine for mandibular blocks. So this very important question remains unanswered.

To summarise, the conclusion reported in this paper that articaine "provides a higher rate of anaesthetic success, with comparable safety to lignocaine when used as infiltration or blocks for routine dental treatments" should be interpreted with caution. Firstly, because individual block and infiltration techniques were not considered in isolation. Secondly, a number of the trials included did not involve any active dental treatment. Articaine has some advantages but these may be dependent upon the particular injection technique and the outcome measure employed.

John Meechan School of Dental Sciences, Newcastle University, Newcastle upon Tyne, UK

1. Mikesell P, Nusstein J, Reader A, Beck M, Weaver J. A comparison of articaine and lidocaine for inferior alveolar nerve blocks. J Endod 2005; 31: 265-270.

2. Kanaa MD, Whitworth JM, Corbett IP, Meechan JG. Articaine and lidocaine mandibular buccal infiltration anesthesia: a prospective randomized double-blind cross-over study. J Endod 2006; 32: 296-298.

3. Abdulwahab M, Boynes S, Moore P, et al. The efficacy of six local anesthetic formulations used for posterior mandibular buccal infiltration anesthesia. J Am Dent Assoc 2009; 140: 1018-1024.

4. Haas DA, Lennon D. Local anesthetic use by dentists in Ontario. J Can Dent Assoc 1995; 61: 319-330.

Evidence-Based Dentistry (2011) 12, 21-22. doi:10.1038/sj.ebd.6400779 